\section{Asthmamanagement bei Kindern}

\section{Welche Inhalationshilfen?}

\author{
Bei der Verordnung einer Asthmamedikation für Kinder wird noch \\ zu wenig Sorgfalt auf die Auswahl der richtigen Inhalationshilfe \\ gelegt. Oberstes Gebot: Die Kinder müssen mit dem System pro- \\ blemlos zurecht kommen und es akzeptieren. Inhalationshilfen der \\ ersten Wahl sind dabei vor allem für kleine Kinder die Spacer.
}

D ie offiziellen Richtlinien der Fachgesellschaften zum Asthmamanagement bei Kindern geben nicht nur Empfehlungen für die einzusetzenden Medikamente, sondern auch für die Auswahl des Inhalationssystems. Doch während zumindest die meisten Ärzte die medikamentöse Behandlung den Richtlinien entsprechend durchführen, herrscht bei der Auswahl der Inhalationssysteme offensichtlich immer noch allgemeine Unsicherheit.

Dies beklagen Ch. O'Callaghan und P. W. Barry in ihrem Editorial im Brit. med. J. vom März dieses Jahres. Gefördert würde diese Unsicherheit durch die Vielzahl der Industrieangebote, so die Autoren. Die Firmen verweisen darüber hinaus in ihren Informationsbroschüren häufig nur auf unpubliziertes Datenmaterial und eine offizielle Zulassung wie bei den Medikamenten gibt es für die Inhalationshilfen nicht. Erst recht existiert keine Übersicht darüber, welche Medikamentendosis das Kind mit welchem Inhalationssystem tatsächlich einatmet.

\section{Kleinkinder brauchen Spacer}

Dabei besteht bei den Fachleuten weitgehend Einigkeit darüber, welche Inhalationssysteme für welche Kinder am besten geeignet sind. So empfehlen die aktuellen Richtlinien aus Großbritannien die Benutzung von Spacern - mit oder ohne Gesichtsmaske - als die Inhalationshilfen der ersten Wahl, vor allem für die Behandlung von Kindern unter 5 Jahren. Ihre Vorteile: Sie sind einfach zu benutzen, die systemische Substanzbelastung bleibt gering, die Behandlungszeiten sind kurz und die Kosten niedrig.
Verweigert das Kind den Spacer, wird auf einen Inhaliergerät zurückgegriffen. Dosieraerosole sind - zumindest bei Kindern unter 6 Jahren - nicht indiziert, da bei ihnen Bedienungsfehler zu oft vorkommen.

Um die Reproduzierbarkeit der eingeatmeten Dosen zu gewährleisten, sollten möglichst antistatische Spacermaterialien wie z.B. Metall gewählt werden. Aber auch die regelmäßige Behandlung mit einem Geschirrspülmittel kann die elektrostatische Aufladung verhindern. Wird der Spacer für die Kortikoidap- plikation verwendet, richtet sich seine Größe nach der zu inhalierenden Dosis.

Bei älteren Kindern bieten sich für die Anwendung von bronchodilatatorisch wirksamen Medikamenten auch die Pulverinhalation oder die atemzuggesteuerte Inhalation eines Dosieraerosols (Autohaler ${ }^{\circledR}$ ) an. Kortikoide können ebenfalls auf diese Weise inhaliert werden, wenn den Kindern die Verwendung eines Spacers zu aufwendig ist.

\section{Weniger ist mehr}

Der Wunsch der Autoren: Die Ärzte sollten sich bei ihrer Verschreibung auf eine Auswahl bestimmter Inhalationshilfen beschränken, mit deren Anwendung sie gut vertraut sind. Und die Eltern sollten sich merken, welches System ihr Kind benutzt, um nicht z.B. in der Notfallambulanz die Ärzte unnötig zu verwirren.

\section{O'Callaghan Ch, Barry PW}

Asthma drug delivery devices for children. Brit med J 2000; 320: 664 .

\title{
Turbohaler: Schulkinder beherrschen ihn
}

\section{Eine Studie zeigt: Fast alle Kinder ab einem Alter von 8 Jahren können nach einer kurzen Einweisung mit einem Pulverinhalator (Turbohaler ${ }^{\circledR}$ ) richtig umgehen.}

\footnotetext{
$\mathrm{D}$ osieraerosole erfreuen sich in der Asthmatherapie großer Beliebtheit - von (kleinen) Kindern werden sie aber kaum richtig bedient und sollten nur in Einzelfällen verordnet werden. Eine Alternative bieten atemzuggesteuerte Pulverinhalatoren wie der Turbohaler. In einer Studie wurde an einem größeren Kollektiv untersucht, wie Kinder verschiedener Altersstufen mit dieser Inhalationshilfe zurecht kommen.

Einbezogen in die Untersuchung waren 161 Kinder mit stabilem Asthma im Alter zwischen 5 und 17 Jahren. Sie wurden 10 Minuten lang mit der richtigen Handhabung des Turbohaler vertraut gemacht.

Nach dieser Einweisung benutzten insgesamt $83 \%$ der Kinder das Gerät korrekt. Aufgeschlüsselt nach Alters-
}

gruppen lag dieser Prozentsatz für Kinder älter als 8 Jahre bei $96 \%$ und für Kinder zwischen 5 und 8 Jahren bei $55 \%$.

Die meisten Kinder, die mit dem Turbohaler Schwierigkeiten hatten, konnten keinen ausreichenden Inspirationsfluss aufbauen.

Eine Kontrolluntersuchung nach etwa 5 Monaten mit 64 Kindern ergab ein ähnliches Ergebnis. Dabei waren nur wenige der Kinder, die bereits zu Anfang Probleme mit dem System hatten, nun in der Lage, den Turbohaler richtig bedienen.

\section{De Boeck $K$ et al.}

Is the correct use of a dry powder inhaler (Turbohaler) age dependent? J Allergy

Clin Immunol 1999; 103: 763-7. 